\title{
Quantitative Trait Loci Analysis of Resistance to Sclerotinia sclerotiorum in Sunflower
}

\author{
S. Rönicke, V. Hahn, A. Vogler, and W. Friedt
}

First, third, and fourth authors: Institute for Crop Science and Plant Breeding, Justus-Liebig-University of Giessen, Heinrich-Buff-Ring 26-

32, D-35392 Giessen, Germany; and second author: State Plant Breeding Institute, University of Hohenheim, D-70593 Stuttgart, Germany. Accepted for publication 2 March 2005.

\begin{abstract}
Rönicke, S., Hahn, V., Vogler, A., and Friedt, W. 2005. Quantitative trait loci analysis of resistance to Sclerotinia sclerotiorum sunflower. Phytopathology 95:834-839.

A quantitative trait loci (QTL) analysis of resistance to Sclerotinia sclerotiorum was carried out with 283 sunflower (Helianthus annuus) $\mathrm{F}_{2: 3}$ families derived from a cross between a resistant (SWS-B-04) and a highly susceptible sunflower inbred line. For that purpose, a genetic map based on 195 amplified fragment length polymorphism and 20 simple sequence repeat markers was constructed. The map has a size of $2,273.5$

centimorgans and comprises 17 linkage groups, 12 of which could be associated to already defined linkage groups. The heads of sunflower $\mathrm{F}_{3}$ families were artificially inoculated by using sclerotinia mycelium in three field environments. The lesion length was measured in centimeters 1 week postinoculation and head rot was scored according to a 1-to-8 head rot scale 2 weeks postinoculation. Using the composite interval mapping procedure, three QTL for lesion length and two QTL for head rot could be identified. These QTL explain 10.6 to $17.1 \%$ of the total phenotypic variance.
\end{abstract}

Sclerotinia root, stem, and head rot are major diseases of sunflower caused by the pertotrophic fungus Sclerotinia sclerotiorum (Lib.) de Bary. There is no efficient means of chemical control of sclerotinia attack, resulting in yield losses of up to $100 \%$ (14). For cultivated sunflower, no pronounced resistance has been identified so far, but quantitative differences in the sclerotinia reaction were described earlier $(4,7,9)$. Also, quantitative trait loci (QTL) analyses of sclerotinia resistance were carried out. Mestries et al. (12) constructed a genetic map with restriction fragment length polymorphism (RFLP) and isoenzyme markers and identified four QTL for leaf resistance and two for head resistance. Simultaneously, Gentzbittel et al. (6) published QTL for resistance to penetration or extension of the $S$. sclerotiorum mycelium in different tissues. The latter authors also mapped a serine-threonine protein kinase-like (PK) locus in the region of one QTL. Later, Bert et al. (2) detected as many as 15 QTL for sclerotinia reaction across several linkage groups, whereas Bert et al. (1) described 7 QTL for resistance to $S$. sclerotiorum terminal bud attack and 4 for capitulum attack. Micic et al. (13) identified nine QTL for leaf lesion, eight for stem lesion, and six for speed of fungal growth. Therefore, a number of QTL could be identified in each of these studies, but the phenotypic variation explained by each of the QTL is mostly small.

It may be difficult to compare the results of different studies on sclerotinia resistance, because the nomenclature of linkage groups used is different. However, it has become possible to compare different genetic maps and the genetic loci for the traits investigated in different studies, because Tang et al. (19) developed simple sequence repeat (SSR) markers, which can be used further as anchors for genetic maps. In a first study, Rachid Al-Chaarani et al. (15) used amplified fragment length polymorphism (AFLP)

Corresponding author: W. Friedt

E-mail address: wolfgang.friedt@ agrar.uni-giessen.de

DOI: 10.1094/PHYTO-95-0834

(C) 2005 The American Phytopathological Society and SSR markers to map QTL for some agronomic traits, but not for sclerotinia resistance. The map comprised 21 groups and a length of 2,915.9 centimorgans (cM).

Only by breeding resistant sunflower lines and hybrids may the control of Sclerotinia rot become possible in the future. Therefore, we studied sclerotinia head reaction of segregating sunflower families and are able to report new results on the genetics of resistance. A simple test for artificial inoculation of sunflower heads using infected millet seeds reported by Rashid (16) had been used effectively by Hahn et al. (7) and Rönicke et al. (18) and, therefore, has been applied in the present work.

The aims of this study were to (i) identify QTL for sclerotinia head resistance of sunflower and (ii) use SSR markers as anchors for an AFLP map to compare our own QTL with those found by other researchers.

\section{MATERIALS AND METHODS}

Plant material. Based on a screening of 25 mostly "high oleic" sunflower inbred lines, one resistant line (SWS-B-04) and a highly susceptible inbred line (SWS-B-01) were identified (17). One $\mathrm{F}_{1}$ plant derived from the cross SWS-B-04 $\times$ SWS-B-01 was self-pollinated to produce $F_{2}$ seed to generate a segregating $F_{2}$ population. Randomly chosen $\mathrm{F}_{2}$ plants were selfed to produce $283 \mathrm{~F}_{3}$ families.

Field experiments. Three field experiments with all $283 \mathrm{~F}_{3}$ families were conducted in 2001: two experiments at Willstätt (Baden), denoted as Willstätt 1 (Wi1) and Willstätt 2 (Wi2), and one experiment at Gross-Gerau (GG), Hesse, Germany. Field trials were conducted in a 29-by-10 triple lattice design (three replications). The $F_{3}$ families were sown on 2 May (GG), 3 May (Wi1), and 18 June (Wi2). The dates of inoculation were 18 July (GG), 25 July (Wi1), and 22 August 2001 (Wi2). Seven plants of each line and each replication were inoculated.

Inoculum and artificial inoculation. Artificial sclerotinia inoculations were performed according to Rashid (16). Sclerotia of $S$. sclerotiorum was grown in petri dishes on malt extract agar 
( $2.0 \%$ malt extract, $1.5 \%$ agar, and $0.2 \%$ peptone) at $25^{\circ} \mathrm{C}$ for 1 to 2 weeks. The same isolate, originating from a single sclerotium collected in 1995 in Eckartsweier (Baden, Germany), was used for all inoculations. A piece of mycelium was cut and transferred to a glass tube containing $30 \mathrm{~g}$ of autoclaved millet (Panicum miliaceum L.) grains and tap water $(40 \mathrm{ml})$. Grains were overgrown with mycelium after 1 to 2 weeks at $25^{\circ} \mathrm{C}$. For artificial inoculation, a small slice was cut into the rear of the sunflower heads with a scalpel. Two grains covered with pathogen mycelium were placed into each cut and taped.

Data recording. Lesion length (i.e., the maximum extension of the lesion beginning from the center of the infection) was measured 1 week after inoculation. Two weeks after inoculation, the symptoms were scored according to a 1-to- 8 head rot scale, where $1=$ lesion length $<1 \mathrm{~cm}, 2=1.0$ to $4.0 \mathrm{~cm}, 3=4.1$ to $8.0 \mathrm{~cm}, 4=$ $25 \%$ of the front infected, $5=50 \%$ of the front infected, $6=75 \%$ of the front infected, $7=100 \%$ of the front infected, and $8=a$ completely destroyed head.

Molecular analysis. Leaf samples from each $\mathrm{F}_{2}$ individual (line) were collected at Gross-Gerau and immediately frozen in liquid nitrogen. Leaf samples were stored at $-20^{\circ} \mathrm{C}$ until DNA extraction. Genomic DNA was isolated from dry frozen leaf material according to Doyle and Doyle (5). AFLP analyses were performed according to Vos et al. (22). The parents of the mapping population were screened with $192 \operatorname{AFLP}($ EcoRI, MseI) and 114 SSR primer combinations for polymorphism. The used 114 primer combinations are the most polymorphic and equally spaced across the sunflower genome primer combinations identified in a large screening of more than 1,000 SSR primer combinations (Z. Micic, University of Hohenheim, personal communication). The $283 \mathrm{~F}_{2}$ lines were analyzed by the most polymorphic 29 AFLP and 26 SSR primer combinations. Polymerase chain reaction conditions for the SSR analyses were according to Tang et al. (19) and $\mathrm{Yu}$ et al. (23). For the AFLP and SSR analyses, infrared fluorescene dye-labeled primer and Licor equipment (MWG Biotech, Ebersberg, Germany) were used.

Field data evaluation. Plants which were not infected (escapes) and did not show symptoms were excluded from the statistical analysis. The percentage infection was calculated as proportion of the infected plants in the total of all inoculated plants. Data from individual $F_{3}$ plants of each subplot were averaged to calculate a subplot mean for each family and replication. Analysis of variance (ANOVA) was performed with field data from each environment. Adjusted entry means and the corresponding error mean squares were used to compute combined analyses of variance across environments. The $\mathrm{F}_{3}$ families and environments were considered as random effects in the ANOVA model. To assess the proportion of the genetic variance versus the total phenotypic variance among entries, heritability estimates $\left(h^{2}\right)$ on an entry mean basis were calculated according to Hallauer and Miranda (8), according to Degener et al. (3). Computations were performed with the computer software package PLABSTAT (20).

Genetic map construction. For construction of the genetic map, the program Mapmaker 3.0 (11) was used. The Kosambi function was applied for map distance calculation (10). First, the "group" command and, after the identification of the groups, the "order" command were used for the localization of AFLP and SSR markers. For markers that could not be integrated with the order command, the "build" and "try" commands were used; only markers which could be located with both orders were used for the genetic map.

QTL-analysis. QTL were detected using the method of composite interval mapping of PLABQTL 1.0 (21). The software and the description can be found on the University of Hohenheim website. To detect the QTL, a minimum log of the likelihood ratio (LOD) score of 2.5 was applied.

\section{RESULTS}

Field experiments. The percentage of infection of the $F_{3}$ families was generally high: 83,99 , and $100 \%$ at Wi1, Wi2, and $\mathrm{GG}$, respectively. The $\mathrm{F}_{3}$ families showed the typical continuous distribution for a quantitative trait, and the inbred parental lines SWS-B-04 and SWS-B-01 could be confirmed as resistant and susceptible (Fig. 1).

The effects of environments and genotypes were highly significant $(P<0.01)$. For head rot the genotype-environment interaction variance was highly significant $(P<0.01)$, and for lesion length it was significant $(P<0.05)$. Heritability values were moderate (i.e., $h^{2}=0.58$ for lesion length and $h^{2}=0.67$ for head rot) (Table 1).

The lesion lengths of the $\mathrm{F}_{3}$ families varied from $0.7 \mathrm{~cm}(\mathrm{Wi1})$ to $8.5 \mathrm{~cm}$ (Wi2), and the average across the three environments was $3.2 \mathrm{~cm}$. For head rot, the rating varied between 2.7 (Wi1) and 7.6 (Wi2), with an average across all environments of 5.2. The resistant inbred line (SWS-B-04) showed a variation from $0.9 \mathrm{~cm}$

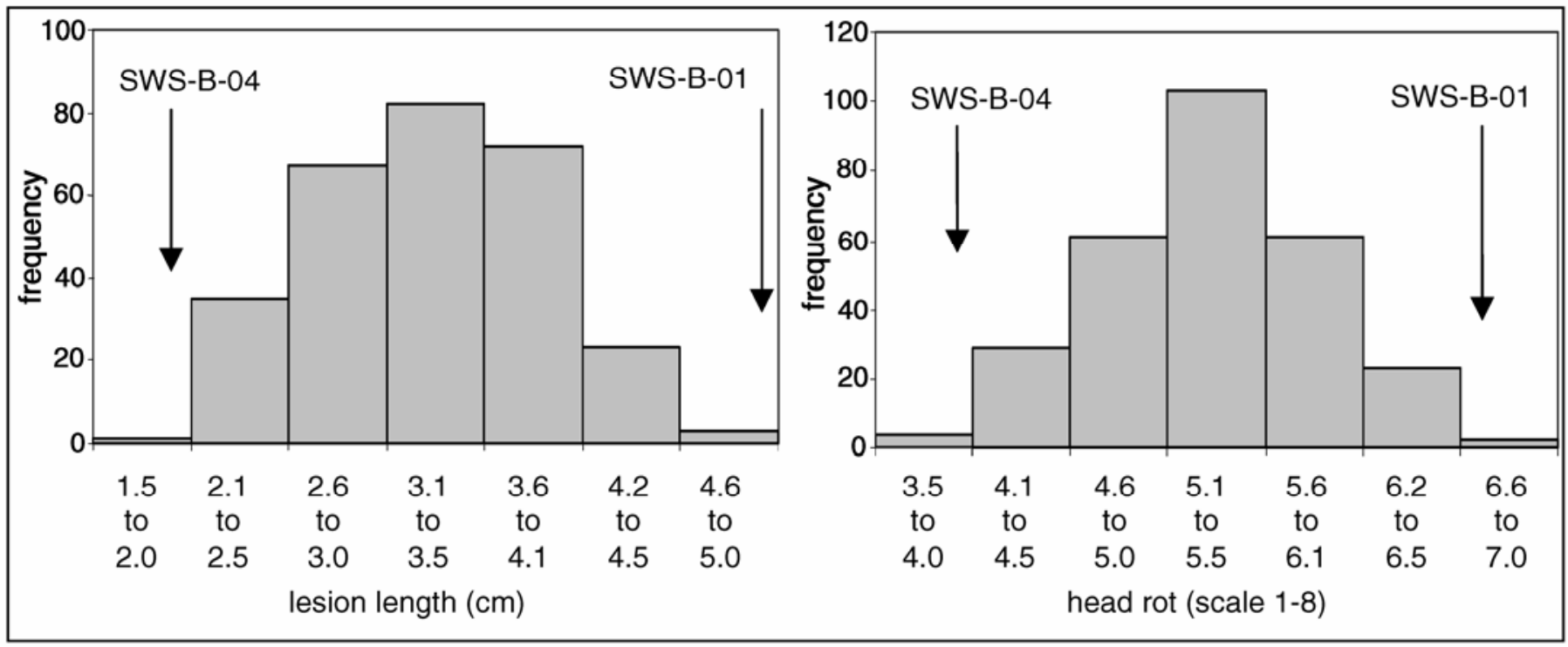

Fig. 1. Frequency distribution for the means of lesion length and head rot of $283 \mathrm{~F}_{3}$ families of the population SWS-B-04 (r) $\times$ SWS-B-01 (s), averaged across three environments. Arrows indicate the mean values of parental inbred lines. 
(Wi1) to $3.0 \mathrm{~cm}(\mathrm{GG})$ for lesion length, whereas the overall average was $2.0 \mathrm{~cm}$. The head rot of SWS-B-04 ranged between 2.9 (GG) and 5.2 (Wi1), with an average across the three environments of 4.0. The lesion lengths of the susceptible parent (SWSB-01) ranged from $3.0 \mathrm{~cm}(\mathrm{GG})$ to $8.5 \mathrm{~cm}$ (Wi2), with a mean for the three environments of $5.3 \mathrm{~cm}$. The head rot of the susceptible inbred line SWS-B-01 varied from 5.6 (Wi1) to 7.6 (Wi2), with an average head rot score of 6.6 (Table 2). A few of the $F_{3}$ families were more susceptible than the susceptible parent, but no one $\mathrm{F}_{3}$ family was significantly better than the resistant parent. The assessments of lesion length and head rot yielded similar results, which showed a fairly high correlation $(r=$ 0.84). In addition, correlations between the sclerotinia results obtained in different environments were all significant and varied from $r=0.27$ for Wi1 versus GG to $r=0.56$ for Wi1 versus Wi2 (Table 3).

Linkage map. A genetic map with 195 AFLP and 20 SSR markers has been constructed (Fig. 2). The map comprises 17 linkage groups (LGs) with an overall size of 2,273.5 cM and an average LG size of $10.6 \mathrm{cM}$. We assume that the developed genetic map represents the whole sunflower genome. Of the 17 groups, 12 could be associated to already defined LGs through the localization of SSR markers $(19,23)$. For these 12 groups, we used the numbering of Tang et al. (19); the other 5 groups were named by letters A to E. The size of the groups varied between 9.2 and $379.9 \mathrm{cM}$, and the numbers of molecular markers per group ranged from 2 to 32 . A maximum of three SSR markers were identified for each linkage group.

QTL detection. For lesion length, three QTL were identified, with LOD scores varying from 2.52 to 3.79 . The explained phenotypic variances of each QTL ranged from 11.3 to $16.3 \%$. QTL on linkage group 10 explained most of the phenotypic variance. The total phenotypic variance explained by the three QTL was $8.6 \%$ and the explained genetic variance was $14.8 \%$.

Two QTL for head rot were identified, which explained 10.6 and $17.1 \%$ of the phenotypic variance at LOD scores of 2.91 and 4.89 , respectively. These two QTL could be associated with defined linkage groups LG1 and LG10 and, together, explained $11.1 \%$ of the phenotypic and $16.6 \%$ of the genetic variance (Table 4). All QTL alleles exhibited additive gene action and the resistance alleles all were contributed by the resistant parent SWS-B-04.

TABLE 1. Estimates of variance components and heritabilities of $283 \mathrm{~F}_{3}$ families for resistance traits evaluated in three environments (Willstätt 1, Willstätt 2, and Gross-Gerau) ${ }^{\mathrm{a}}$

\begin{tabular}{lccc}
\hline Source of variance & df & $\begin{array}{c}\text { Lesion length } \\
(\mathrm{cm})\end{array}$ & $\begin{array}{c}\text { Head rot } \\
(\text { scale 1 to 8) }\end{array}$ \\
\hline Environment & 2 & $0.266^{* *}$ & $0.211^{* *}$ \\
Genotype & 282 & $0.194^{* *}$ & $0.220^{* *}$ \\
Genotype $\times$ environment & 564 & $0.052^{* *}$ & $0.088^{* *}$ \\
Error & 1,218 & 1.086 & 0.726 \\
$h^{2}$ & $\ldots$ & 0.58 & 0.67 \\
\hline a $*$ and $* *=$ significant at $P<0.05$ and $<0.01$, respectively. &
\end{tabular}

\section{DISCUSSION}

For artificial sclerotinia infection, the method of Rashid (16) was used in the present experiments. The high percentage of infection under the three field environments and the highly significant genotype variance indicate that this method can be used efficiently in screening sunflower materials for sclerotinia resistance. Similar to an earlier study, the inbred SWS-B-04 was confirmed as resistant and SWS-B-01 as highly susceptible (17).

The correlations between environments were significant but moderate. Nevertheless, the genotype-environment interaction variance was always smaller than the genotypic variance. The lesion length measurements and head rot scores were closely related and the heritability estimates were similar for both traits. Consequently, to assess one of these traits would be sufficient to screen for sclerotinia resistance of the sunflower head in a breeding program. We would recommend the lesion length measurement, because it is easier and faster than scoring head rot.

The genetic map based on 195 AFLP and 20 SSR markers has a size of 2,273.5 cM. In comparison, Bert et al. $(1,2)$ constructed genetic maps with sizes of 2,318 and 1,937 cM. In contrast, Mestries et al. (12) built a genetic map with a size of $760 \mathrm{cM}$ only, but the authors exclusively used codominant markers (RFLPs, isoenzymes). The SSR maps of Tang et al. (19) $(1,368.3$ cM), Yu et al. (23) (1,199.4 to 1,423.0 cM), and Micic et al. (13) $(941.4 \mathrm{cM})$ also were smaller than the maps of Bert et al. $(1,2)$ and the present map. These differences may be due to the use of dominant markers, as was the case with the latter studies.

We used the SSR markers of Tang et al. (19) to identify LGs 1, $4,5,6,7,8,9,10,11,12,16$, and 17 . On LG 10, we identified one QTL for lesion length and one QTL for head rot. Interestingly, Mestries et al. (12) detected QTL for the reaction of capitula after inoculation with mycelium explants on their group A and Bert et al. $(1,2)$ found QTL on their group 7, both of which are equivalent to LG 10 in the present study (A. Leon, Advanta Semillas, Balcarce Research Station, Argentina; personal communication).

It is very interesting to note that, in different sunflower mapping populations, QTL involved in the reaction of the capitulum against mycelium extension were found on the same linkage group. Bert et al. $(1,2)$ infected the sunflower heads with ascospores and also identified further QTL for resistance of the capitulum on group 7. Mestries et al. (12) identified QTL on group A for the sclerotinia reaction as expressed by the lesion length on artificially inoculated leaves.

TABLE 3. Correlations $(r)$ between environmental data for lesion length and head rot score of $283 \mathrm{~F}_{3}$ families ${ }^{\mathrm{a}}$

\begin{tabular}{lcc}
\hline Location & Lesion length & Head rot \\
\hline Willstätt1:Willstätt2 & $0.50^{* *}$ & $0.56^{* *}$ \\
Willstätt1:Gross-Gerau & $0.34^{* *}$ & $0.27^{* *}$ \\
Willstätt2:Gross-Gerau & $0.39^{* *}$ & $0.46^{* *}$ \\
\hline
\end{tabular}

a $* *=$ significant at $P<0.01$.

TABLE 2. Results of the field experiments after artificial inoculation with Sclerotinia sclerotiorum at Willstätt 1, Willstätt 2, and Gross-Gerau

\begin{tabular}{|c|c|c|c|c|}
\hline \multirow[b]{2}{*}{ Genotype or population } & \multirow[b]{2}{*}{ Overall average } & \multicolumn{3}{|c|}{ Average (minimum to maximum) } \\
\hline & & Willstätt 1 & Willstätt 2 & Gross-Gerau \\
\hline \multicolumn{5}{|l|}{ Lesion length } \\
\hline $\mathrm{F}_{3}$ & 3.2 & $2.7(0.7$ to 5.1$)$ & $3.4(0.8$ to 8.5$)$ & $3.7(2.5$ to 4.5$)$ \\
\hline SWS-B-04 & 2.0 & $1.4(0.9$ to 1.7$)$ & $1.7(0.8$ to 2.3$)$ & $2.8(2.5$ to 3.0$)$ \\
\hline SWS-B-01 & 5.3 & $4.7(4.0$ to 5.1$)$ & $7.3(6.1$ to 8.5$)$ & $3.9(3.0$ to 4.0$)$ \\
\hline \multicolumn{5}{|l|}{ Head rot } \\
\hline $\mathrm{F}_{3}$ & 5.2 & $5.0(2.7$ to 6.8$)$ & $4.9(2.9$ to 7.6$)$ & $5.7(2.9$ to 7.5$)$ \\
\hline SWS-B-04 & 4.0 & $4.8(4.5$ to 5.2$)$ & $3.5(3.4$ to 3.5$)$ & $3.9(2.9$ to 4.8$)$ \\
\hline SWS-B-01 & 6.6 & $6.1(5.6$ to 6.7$)$ & $6.9(6.4$ to 7.6$)$ & $6.9(6.7$ to 7.1$)$ \\
\hline
\end{tabular}


LG 1

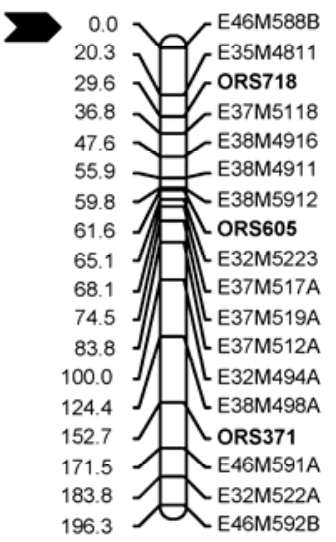

LG 4

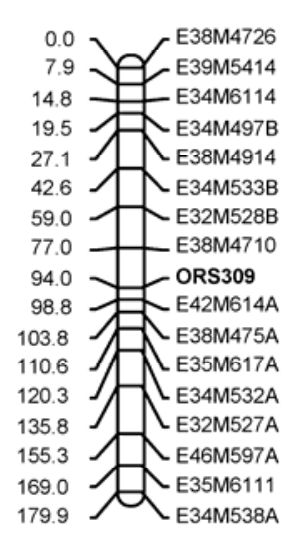

LG 5

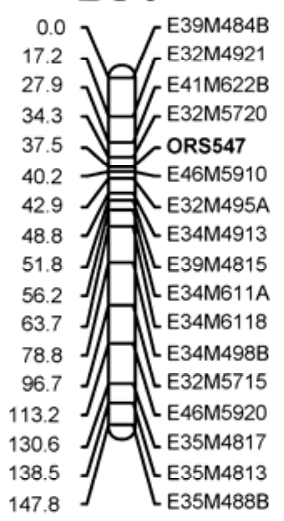

LG 6

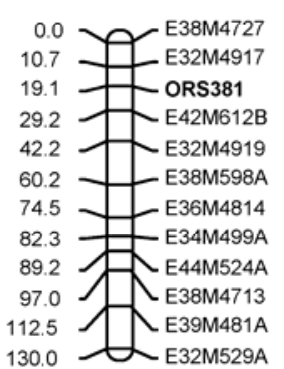

LG 7

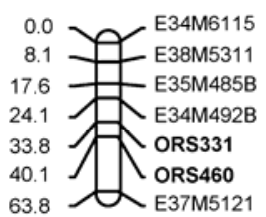

\section{LG 8}

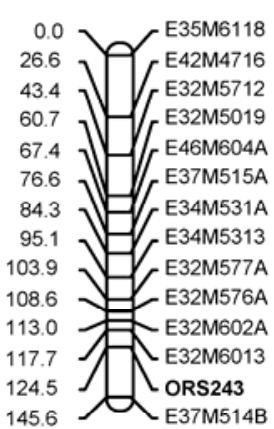

LG A

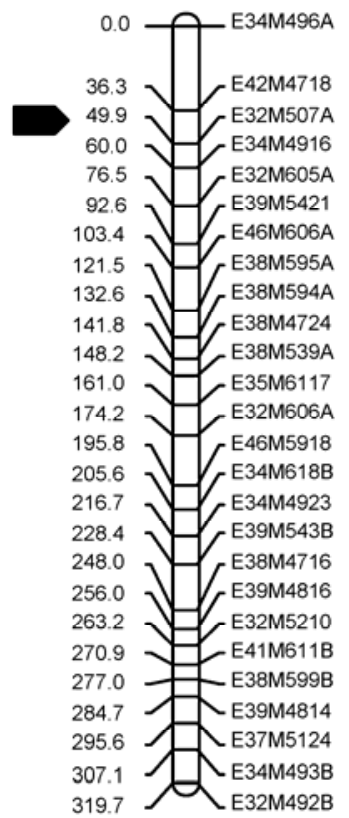

LG 9

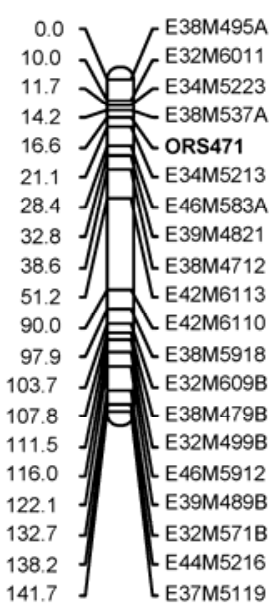

LG 10

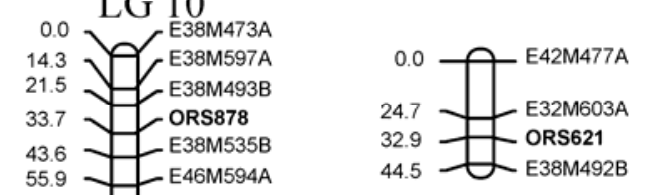

LG 12

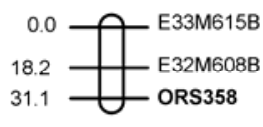

\section{LG 17}

LG 16

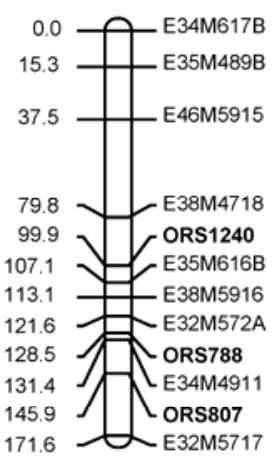

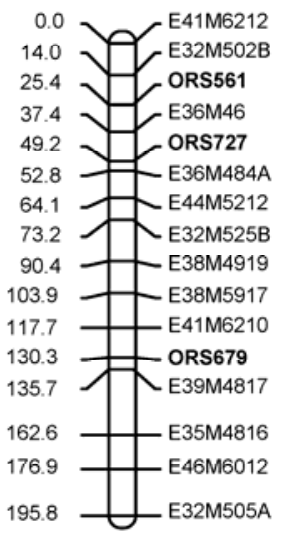

LG B

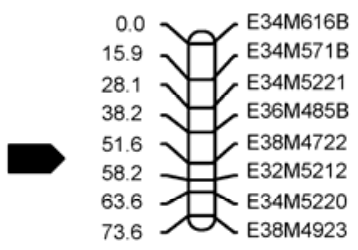

LG C

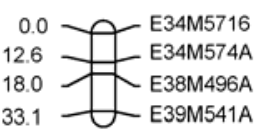

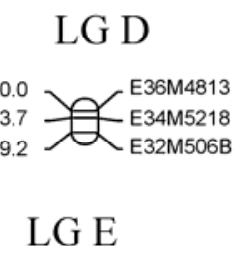

0.0 E32M4913

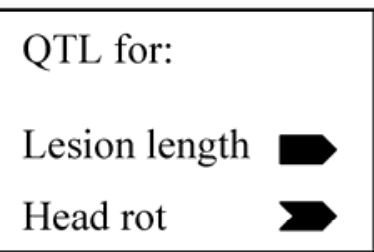

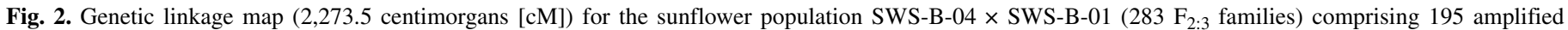

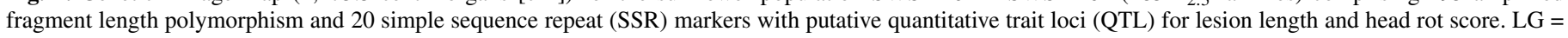
linkage groups, distances are given in centimorgans, SSR markers are printed in bold, and QTL are marked by symbols as indicated in the box. 
TABLE 4. Parameters associated with putative quantitative trait loci (QTL) for two resistance traits estimated from genotypic and phenotypic data of $283 \mathrm{~F}_{2: 3}$ families of the cross SWS-B-04 $\times$ SWS-B-01 evaluated in three environments (Gross-Gerau, Willstätt 1, and Willstätt 2)

\begin{tabular}{|c|c|c|c|c|c|c|}
\hline Sclerotinia reaction trait & LG & $\begin{array}{l}\text { Position on LG } \\
\text { (cM) }\end{array}$ & Marker interval & $\begin{array}{l}\text { LOD at QTL } \\
\text { position }\end{array}$ & $\begin{array}{l}\text { Additive genetic } \\
\text { effect }(\%)\end{array}$ & $\begin{array}{l}\text { Phenotypic variance } \\
\text { explained }(\%)\end{array}$ \\
\hline \multirow{2}{*}{ Lesion length } & LG 10 & 304 & E35M6114-E38M534A & 3.79 & 0.322 & 16.3 \\
\hline & LG A & 52 & E32M507A-E34M4916 & 2.88 & 0.327 & 12.4 \\
\hline Total & $\ldots$ & $\ldots$ & $\ldots$ & $\ldots$ & $\ldots$ & 8.6 \\
\hline \multirow[t]{2}{*}{ Head rot } & LG 1 & 6 & E46M588B-E35M4811 & 4.89 & 0.398 & 17.1 \\
\hline & LG 10 & 356 & E39M544B-E46M584B & 2.91 & 0.356 & 10.6 \\
\hline Total & $\ldots$ & $\ldots$ & $\ldots$ & $\ldots$ & $\ldots$ & 11.1 \\
\hline
\end{tabular}

${ }^{a} \mathrm{LG}=$ linkage group, $\mathrm{cM}=$ centimorgans, $\mathrm{LOD}=\log$ of the likelihood ratio.

One QTL was identified for head rot on LG 1. This is consistent with Bert et al. (2) who identified QTL for mycelium on heads and leaves and percentage head attack on the same group (A. Leon, personal communication). Mestries et al. (12) detected QTL for the reaction of capitula after inoculation with mycelium explants (capitulum index) on this group, too. Micic et al. (13) also located one QTL for leaf lesion and one for speed of fungal growth on LG 1.

Bert et al. $(1,2)$ identified QTL for the reaction of the sunflower head which explained 9.0 to $19.9 \%$ and 2.51 to $19.84 \%$, respectively, of the phenotypic variance. In the present study, the detected QTL explain a similar part of the phenotypic variance (10.6 to $17.3 \%$ ). We found no QTL partially explaining the variance of both traits, but lesion length and head rot is highly positive correlated $(r=0.84)$. Because of this correlation, the same or similar resistance genes may be involved, but we found QTL on four different linkage groups and the two QTL for different versions of the disease on LG 10 were not closely linked. Furthermore, it is most likely that not all genetic factors involved in sclerotinia head resistance of sunflower were identified with the QTL analysis presented here. This is indicated by the comparatively small part of genotypic and phenotypic variances explained.

Up to now, marker-assisted selection (MAS) for sclerotinia resistance is not yet possible because of the lack of molecular markers. For the development of such closely linked markers, further studies are necessary. We propose that QTL identified in different independent studies are good candidates to develop markers for MAS. To analyze whether the same or different QTL are involved in sclerotinia reaction, identical markers but different sunflower populations and different inoculation methods should be used. Both LG 1 and LG 10 are very good candidates for further analyses to develop molecular markers for sclerotinia reaction, because QTL for sclerotinia resistance could be identified on these two groups in a number of different independent studies.

\section{ACKNOWLEDGMENTS}

Financial support of the project FNR ÖE 115/00 NR (99 NR 055) was provided by the Bundesministerium für Verbraucherschutz, Ernährung und Landwirtschaft (BMVEL) and the Gemeinschaft zur Förderung der privaten deutschen Pflanzenzüchtung e.V. (GFP), Bonn, Germany. We thank J. Döhrel, S. Schillinger, and M. Tolksdorf for excellent technical assistance.

\section{LITERATURE CITED}

1. Bert, P. F., Dechamp-Guillaume, G., Serre, F., Jouan, I., Tourvieille de Labrouhe, D., Nicolas, P., and Vear, F. 2004. Comparative genetic analysis of quantitative traits in sunflower (Helianthus annuus L.) 3. Characterisation of QTL involved in resistance to Sclerotinia sclerotiorum and Phoma macdonaldi. Theor. Appl. Genet. 109:865-874.

2. Bert, P. F., Jouan, F., Tourvieille de Labrouhe, D., Seere, F., Nicolas, P., and Vear, F. 2002. Comparative genetic analysis of quantitative traits in sunflower (Helianthus annuus L.) 1. QTL involved in resistance to Sclerotinia sclerotiorum and Diaporthe helianthi. Theor. Appl. Genet. 105:985-993.

3. Degener, J., Melchinger, A. E., Gumber, R. K., and Hahn, V. 1998. Breeding for Sclerotinia resistance in sunflower: A modified screening test and assessment of genetic variation in current germplasm. Plant Breed. 117:367-372.

4. Degener, J., Melchinger, A. E., and Hahn, V. 1999. Optimal allocation of resources in evaluating current sunflower inbred lines for resistance to Sclerotinia. Plant Breed. 118:157-160.

5. Doyle, J. F., and Doyle, J. L. 1990. Isolation of plant DNA from fresh tissue. Focus 12:13-15.

6. Gentzbittel, L., Mouzeyar, S., Badaoui, S., Mestries, E., Vear, F., Tourvieille De Labrouhe, D., and Nicolas, P. 1998. Cloning of molecular markers for disease resistance in sunflower, Helianthus annuus L. Theor. Appl. Genet. 96:519-525.

7. Hahn, V. 2002. Genetic variation for resistance to Sclerotinia head rot in sunflower inbred lines. Field Crops Res. 77:153-159.

8. Hallauer, A. R., and Miranda, J. B 1981. Quantitative Genetics in Maize Breeding. Iowa State University Press, Ames.

9. Köhler, H., and Friedt, W. 1999. Genetic variability as identified by APPCR and reaction to mid-stem infection of Sclerotinia sclerotiorum among interspecific sunflower (Helianthus annuus L.) hybrid progenies. Crop Sci. 39:1456-1463.

10. Kosambi, D. D. 1944. The estimation of map distances from recombination values. Ann. Eugen. 12:172-175.

11. Lander, E. S., Green, J., Abrahamson, J., Batlow, A., Daly, M. J., Lincoln, S. E., and Newburg, L. 1987. MAPMAKER: An interactive computer package for constructing primary genetic linkage maps of experimental and natural populations. Genomics 1:174-181.

12. Mestries, E., Gentzbittel, L., Tourvieille de Labrouhe, D., Nicolas, P., and Vear, F. 1998. Analysis of quantitative trait loci associated with resistance to Sclerotinia sclerotiorum in sunflowers (Helianthus annuus L.) using molecular markers. Mol. Breed. 4:215-226.

13. Micic, Z., Hahn, V., Bauer, E., Schön, C., Knapp, S. J., Tang, S., and Melchinger, E. 2004. QTL mapping of Sclerotinia midstalk rot resistance in sunflower. Theor. Appl. Genet. 109:1474-1484.

14. Péres, J., and Regnault, Y. 1985. Sclerotinia sclerotiorum (Lib.) de Bary: Recherche de moyens chimiques permettant de limiter la production d'inoculum par traitement du sol. Pages 363-368 in: Proc. 11th Int. Sunflower Conf. Mar del Plata, Argentina.

15. Rachid Al-Chaarani, G., Gentzbittel, L., Huang, X. Q., and Sarrafi, A. 2004. Genotypic variation and identification of QTLs for agronomic traits, using AFLP and SSR markers in RILs of sunflower (Helianthus annuus L.). Theor. Appl. Genet. 109:1353-1360.

16. Rashid, K. 1997. Inoculation methods for the assessment of Sclerotinia head rot in sunflower. Pages 72-75 in: Proc. 19th Sunflower Res. Workshop, Fargo, ND.

17. Rönicke, S., Hahn, V., and Friedt, W. Resistance to Sclerotinia sclerotiorum of "high oleic" sunflower inbred lines. Plant Breed. (In press.)

18. Rönicke, S., Hahn, V., Horn, R., Gröne, I., Brahm, L., Schnabl, H., and Friedt, W. 2004. Interspecific hybrids of sunflower as a source of Sclerotinia resistance. Plant Breed. 123:152-157.

19. Tang, S., Yu, J. K., Slabaugh, M. B., Shintani, D. K., and Knapp, S. J. 2002. Simple sequence repeat map of the sunflower genome. Theor. Appl. Genet. 105:1124-1136.

20. Utz, H. F. 2000. PLABSTAT: A computer program for statistical analysis of plant breeding experiments. Institute for Plant Breeding, Seed Science and Population Genetics, University of Hohenheim, Stuttgart, Germany.

21. Utz, H. F., and Melchinger, A. E. 1996. PLABQTL: A program for 
composite interval mapping of QTL. J. Quant. Trait Loci 2(1).

22. Vos, P., Hogers, R., Bleeker, M., Reijans, M., van de Lee, T., Hornes, M.,

Frijters, A., Pot, J., Peleman, J., Kuiper, M., and Zabeau, M. 1995.

AFLP: A new technique for DNA fingerprinting. Nucleic Acids Res. 23:4407-4414.
23. Yu, J. K., Tang, S., Slabaugh, M. B., Heesacker, A., Cole, G., Herring, M., Soper, J., Han, F., Chu, W. C., Webb, D. M., Thompson, L., Edwards, K. J., Berry, S., Leon, A., Olungu, C., Maes, N., and Knapp, S. J. 2003. Towards a saturated molecular genetic linkage map for cultivated sunflower. Crop Sci. 43:367-387. 